Neurosurg Focus 13 (6):Article 1, 2002, Click here to return to Table of Contents

\title{
Bone healing and spinal fusion
}

\author{
Julie G. Pilitsis, M.D., Ph.D., David R. Lucas, M.D., and Setti R. Rengachary, M.D. \\ Department of Neurosurgery, Wayne State University, Detroit, Michigan
}

\begin{abstract}
Bone is a tissue that constantly undergoes deposition, resorption of stromal matrix, and remodeling. These processes may be altered by a variety of chemical, mechanical, cellular, and pathological mechanisms. Understanding the physiology of bone healing and the mechanisms affecting this process is important not only when evaluating normal skeletal development but also when initiating fracture repair. Because the ultimate success of spinal fusions involves creation of an osseous union, we focus this review on the anatomy and physiology of bone under physiological conditions, normal bone healing and mechanisms that alter it, and available adjuvant therapies that may enhance healing potential in a clinical setting.
\end{abstract}

KEY WORDS - bone healing • bone graft - bone morphogenetic protein - spinal fusion

Bone is unique tissue because it is continuously metabolically active and thus subject to a variety of systemic and local factors throughout life. Clinicians and researchers have attempted to optimize the process of bone healing in the treatment of fractures and in the creation of successful bone fusions. The creation of adequate spinal fusions is of considerable interest to neurosurgeons and orthopedic spine surgeons, because unsuccessful spinal fusions result in significant morbidity and often reoperation. Although temporary support in spinal arthrodeses is achieved with the placement of instrumentation, permanent support is achieved through creation of a solid osseous union. To allow for a successful bone union, clinicians must understand the physiology of bone healing, the roles of regulators and inhibitors in this process, and the proper use of materials that may promote bone healing in vivo.

\section{CELLULAR COMPONENTS OF BONE}

Bone consists of the following cell types: osteogenic precursor cells, osteoblasts, osteocytes, osteoclasts, and hemopoietic bone marrow cells. ${ }^{14,40}$ Osteogenic precursor cells are stem cells derived from mesenchyme and have the potential to undergo mitosis and differentiation into

Abbreviations used in this paper: $\mathrm{BMP}=$ bone morphogenetic protein; $\mathrm{DBM}=$ demineralized bone matrix; HA = hydroxyapatite; PDGF = platelet-derived growth factor; PTH = parathyroid hormone; rhBMP = recombinant human $\mathrm{BMP} ; \mathrm{TGF}=$ transforming growth factor. mature cells. ${ }^{20}$ These cells are present on the deep periosteal layer and on the endosteal layer that lines the internal medullary surfaces. ${ }^{31}$

Osteoblasts, the bone-forming cells, are involved in matrix development as well as calcification. They have receptors for a variety of hormones, vitamins, and cytokines and are believed to be involved both in bone deposition and resorption by activating osteoclasts. As the process of bone deposition ends, some osteoblasts remain on the periosteum and endosteum, whereas others become osteocytes. ${ }^{31}$ Woven bone, present in embryonic development and in fracture healing, contains osteoid, which is unmineralized bone matrix and mineralized matrix formed by irregularly placed collagenous fibers. ${ }^{31}$ The intervening stroma consists of reactive fibrovascular tissue. With bone maturation, osteoid is distributed in a concentric fashion forming lamellae. ${ }^{20}$ In cancellous bone, continued osteoblastic activation results in the calcification of the trabeculae of bone matrix; however, only limited thickening of this matrix occurs. In bone destined to become compact bone, osteoblastic-induced thickening of the matrix continues and lamellae demonstrate more organization than in woven bone and tighter packing than in cancellous bone. ${ }^{31}$

Osteocytes are created when mature osteoblasts become entrapped within the osseous matrix. These cells are involved in the control of extracellular concentrations of $\mathrm{Ca}$ and phosphate. Each osteocyte has a number of branching processes that extend through canaliculi to allow for cell-to-cell interaction and passage of ions and growth factors from blood vessels to osteocytes. Gap junctions between processes allow for passage of biologi- 
cally active molecules. In compact bone, osteons or haversian systems are present, and the strength of the bone depends on their presence. ${ }^{20}$ Each consists of five to 20 lamellae surrounding a haversian canal containing a blood vessel. ${ }^{31}$ Horizontal canals, known as Volkmann canals, connect osteons and allow for cellular communication and blood vessels from the periosteum to cross the matrix.

Osteoclasts are multinucleated macrophages that secrete proteolytic enzymes. These cells acts in groups and are able to dissolve both the inorganic and organic osseous matrices, resulting in the formation of erosive pits called Howship lacunae and the release of $\mathrm{Ca}$ and phosphate. ${ }^{31}$ They have receptors for calcitonin, which, when activated, suppress resorption. They do not, however, have PTH receptors. ${ }^{20}$ Parathyroid hormone activates receptors on osteoblasts, and osteoblasts subsequently release osteoclaststimulating factor that then activates osteoclasts to begin resorption. ${ }^{20}$ Osteoclasts disappear after resorption.

\section{BONE MATRIX}

As described in the previous section, bone consists both of cells and intercellular matrix. Twenty percent of bone is water. ${ }^{40}$ The dry weight consists of approximately 30 to $35 \%$ organic matrix and 65 to $70 \%$ inorganic substances. ${ }^{31}$ The organic component consists of collagenous fibers and glycosaminoglycans, such as chondroitin sulfate, hyaluronic acid, and keratan sulfate, which provide the tissue's strength. ${ }^{20}$ Type I collagen is the prominent collagen in bone and has a greater number of intermolecular bonds than other collagen types, which may lead to its increased strength and inability to swell. ${ }^{20}$ Also present are the osteoblast products osteocalcin and osteopontin that bind to $\mathrm{HA}$, stimulate release of the active vitamin $\mathrm{D}$ metabolite, $1 \alpha 25$-dihydroxycholecalciferol, and are possibly involved in the binding of osteoblasts and osteoclasts to bone..$^{20}$ Osteonectin has been identified as a stimulator of vitamin $\mathrm{D}$ metabolism, although its complete function remains unclear. ${ }^{20}$ The inorganic matrix consists of approximately $85 \% \mathrm{Ca}$ phosphate, $10 \% \mathrm{CaCO}_{2}, 5 \%$ fluoride derivatives including $\mathrm{Ca}$ fluoride and magnesium fluoride, and trace elements. ${ }^{20}$ Calcium phosphate is present in the form of $\mathrm{HA}$, and this substance is deposited in pores between the collagenous fibers during the process of mineralization. The initial mineralization of osteoid occurs within days of secretion but may continue for months. ${ }^{31}$ The inorganic substances provide hardness and rigidity to bone tissue. The relative relation of these inorganic substances to organic matrix increases with aging and decreases because of rickets and osteomalacia. ${ }^{20}$

\section{HORMONAL REGULATION OF BONE METABOLISM}

Bone metabolism is regulated by hormones and growth factors. The most influential hormones in this process are PTH, calcitonin, and vitamin D, although other vitamins, electrolytes, and hormones also affect bone metabolism. The release of PTH occurs in response to a decrease in plasma concentration of $\mathrm{Ca}$. This hormone acts on osteoblasts and leads to the release of osteoclast-stimulating factor, which activates the resorption process. ${ }^{20}$ It may also promote enhanced renal absorption of $\mathrm{Ca}$ and stimulation of renal production of $1 \alpha 25$-dihydrocholecalciferol, thereby increasing intestinal $\mathrm{Ca}$ absorption. ${ }^{20}$ When administered in intermittent low doses, PTH has been shown both in animals and humans to stimulate bone growth, and Phase III clinical trials are currently in progress to assess the efficacy of the osteogenic component of PTH hPTH(1-34) in treatment of osteoporosis. ${ }^{37}$ Furthermore, new PTH analogs have been synthesized that cause less hypercalcemia. ${ }^{37}$

Calcitonin is secreted by the parafollicular cells of the thyroid gland in response to rises in plasma Ca concentrations and is involved in the inhibition of $\mathrm{Ca}$-dependent cellular processes. Calcitonin has been recognized to be useful in the treatment of osteopenia and osteoporosis. In clinical studies, the rate of fracture per 100 patient years was 6.94 and 16.56 for calcitonin- and noncalcitonintreated patient groups. ${ }^{32}$ Furthermore, in one case report the authors described the successful fusion of an odontoid fracture in a patient who underwent immobilization in a Philadelphia collar and an eight-week course of nasal calcitonin therapy. ${ }^{16}$

Vitamin D stimulates increased renal and intestinal absorption of Ca. Vitamin D deficiency results in decreases in absorption and lower phosphate plasma concentrations. $^{20}$ This deficiency causes rickets in children and osteomalacia in adults. ${ }^{20}$ Vitamin $\mathrm{C}$ deficiency retards collagen synthesis, results in impaired maintenance of osteocollagenous fibers, and ultimately inhibits proper bone formation. ${ }^{20}$ Vitamin A deficiency results in impaired osteoblastic production of osteoid and impaired resorption whereas excessive vitamin A leads to an increase in osteoclasts. $^{20}$

Other regulators of bone metabolism include growth hormone and anabolic steroids. Growth hormone is secreted by the anterior hypophysis of the pituitary and stimulates cellular division in cartilage. Oversecretion causes bone deposition in excess of resorption and ultimately leads to gigantism, whereas undersecretion leads to dwarfism. ${ }^{20}$ Anabolic steroids have been shown in animal models to induce osteogenesis. Administration of testosterone, dihydrotestosterone, and androstendione in rats resulted in increases in cortical bone. ${ }^{49}$ Currently, systemic administration continues to results in virilizing side effects. ${ }^{49}$ Although hormone replacement therapy is currently controversial and is beyond the scope of this review, $17 \beta$ - estradiol has been shown to increase osteoblastic proliferation and collagen synthesis in vitro. ${ }^{20}$ Furthermore, there is evidence that in osteoporotic patients treated with hormone replacement therapy, higher bone mineral density is present than in those treated with bisphosphates or calcitonin. ${ }^{48}$

\section{ROLE OF GROWTH FACTORS IN BONE METABOLISM}

Growth factors are released from platelets, macrophages, and fibroblasts and include BMP, TGF, PDGF, fibroblastic growth factors, and insulin-like growth factors. They are able to induce stem cell differentiation and chemotaxis, vascularization, and osteoinduction. In the 1960s, Urist ${ }^{50}$ first demonstrated that ectopically implant- 
ed DBM induced bone formation. Subsequent molecular biological investigations reported that the essential component in DBM allowing for osteoinduction was BMP. ${ }^{12,53}$ The BMPs have been shown to play crucial roles in normal skeletal development as well as bone healing and are able to activate transcription of genes involved in cellular migration, proliferation, and differentiation. ${ }^{18}$ The delivery of BMP on matrices has been efficacious in the treatment of long bone fractures and spinal fusion..$^{9,22,23}$ Studies involving the introduction of BMP-2 complimentary DNA introduction in animal models are underway. ${ }^{22,25}$ Adenovectors containing BMP-2, $-4,-6$, and -9 have been shown to be osteogenic in rodents ${ }^{1,2,52}$ and heterodimers such as BMP-4/7 and BMP-2/7 demonstrate increased osteogenesis compared with homodimers. ${ }^{28,41}$ Immune responses currently limit osteogenesis in these models. ${ }^{22}$ Alternatively recombinant BMPs (rhBMP-2 and rhBMP7) have also been developed and have been successful in spinal fusion.

In the 1990s, several groups evaluated spinal fusion rates when using rhBMP-2 in various concentrations and in various carriers compared with autologous bone grafts in animal models. ${ }^{6-8,25,42}$ They found higher rates of fusion and increased biomechanical stability of these fusions. These findings prompted nonhuman primate studies, in which the investigators confirmed successful fusions. The authors of a recent pilot study in humans reported successful radiographic and clinical arthrodeses at 6 months in all patients who underwent anterior lumbar interbody fusion and decreased hospital stay compared with patients treated with autograft-filled cages. ${ }^{6}$ In addition, the authors of animal studies have shown that rhBMP-2 is similarly effective as an osteoinductive agent in irradiated and nonirradiated tissue, thus providing hope for the complex task of fusion in the patient who undergoes radiotherapy. ${ }^{27}$ In the future BMPs will have a major role in spinal fusions. 22

Although BMP appears the most osteogenic of the polypeptides, it is important to mention TGF $\beta$ and PDGF as other potential osteoinductive agents in spinal fusion. ${ }^{22}$ Platelets have high levels of TBF $\beta$ and PDGF ${ }^{36}$ In addition, TGF- $\beta$ is present in bone and is released from platelets after injury. ${ }^{17}$ It stimulates osteogenesis, angiogenesis, fibroblast migration, and deposition of matrix, although to a lesser degree than BMP. ${ }^{22}$ Additionally PDGF stimulates osteoblast proliferation, collagen synthesis, and may play a regulatory role in fracture repair. ${ }^{22,33}$ It is normally present at fracture sites and, depending on its polypeptide chains, may act systemically or locally. ${ }^{3,22}$ In a model of osteotomy in rabbit, PDGF on a collagen carrier was demonstrated to increase osteogenesis and callus formation compared with animals treated with collagen alone. ${ }^{38}$

\section{REPAIR OF BONE}

There are three main phases following fracture in the bone repair process: 1 ) the early inflammatory stage; 2 ) the repair stage; and 3 ) the remodeling stage. ${ }^{11,17}$ The early inflammatory phase comprises the first 2 weeks postinjury and is initiated after hemorrhage caused by vascular injury and the subsequent development of a hematoma. Infiltration of inflammatory cells and of fibroblasts into the area then occurs (Fig. 1 upper). These events lead to vascularization of the area and the formation of granulation tissue (that is, procallus). The repair phase is characterized by the formation of a callus. It begins with continued vascular ingrowth, secretion of osteoid, and the presence of fibrocollagenous fibers. A temporary callus consisting of cartilage is produced at the site of injury (Fig. 1 center). This initial union develops in the first 4 to 6 weeks and has limited strength; thus, internal/external immobilization of the fracture/fusion site is often appropriate..$^{31}$ Osteoblasts continue to be active and replace cartilage with the cancellous bone forming a bridge between the fractured fragments (Fig. 1 lower). Cancellous bone may then be converted to compact bone as osteoid deposition continues. In the remodeling phase the process may occur over months to years and consists of restoring the fractured bone to its normal size, shape, and strength. ${ }^{31}$ Adequate strength usually develops by 6 months.

A variety of factors may limit proper bone healing. The early inflammatory stage may be adversely altered by the administration of antiinflammatory and cytotoxic medications and of steroid agents. ${ }^{19,43,46}$ Vascularization of the tissue and overall healing in the first few weeks is often impaired by nicotine..$^{15}$ Radiation and systemic illnesses such as diabetes mellitus, rheumatoid arthritis, and osteoporosis are recognized inhibitors of successful bone healing and fusion. ${ }^{31}$ Advanced age also limits an individual's healing potential. For example, the rate of bone loss increases from 0.3 to $0.5 \%$ a year before menopause and to 2 to $3 \%$ after menopause. ${ }^{20}$ Furthermore, the number of bone marrow stem cells drops considerably during aging. An infant has one stem cell per 10,000 marrow cells whereas an elderly patient has one stem cell in 2 million marrow cells. ${ }^{44} \mathrm{~A}$ combination of factors therefore must be considered when attempting to induce a successful fusion in older patients.

Biomechanical concerns must also be carefully considered in the bone healing process during fusion. It has been appreciated for more than a century that bone forms in places where stress requires its presence. ${ }^{40}$ The proper amount of stress placed on a graft varies based on anatomical region and overall stability of the bone. ${ }^{34}$ Resorption occurs in areas where bone is not exposed to mechanical stresses. ${ }^{31}$ It is important when using bone grafts in fusion operations to optimize the mechanical stresses placed on the graft. Bed rest, the placement of rigid instrumentation, and brace therapy may shield the bone from mechanical stress and result in resorption and weakening of the graft. ${ }^{34}$ Other biomechanical concerns in bone graft use include the fit between the graft and host bone, the amount of surface area contact between the two bones, and the characteristics of the contact surfaces. ${ }^{34}$

\section{BONE GRAFTS}

The ability of bone to heal and of fusions to form is based on three key concepts: osteogenesis, osteoinduction, and osteoconduction. Osteogenesis, defined as the ability to produce new bone, is determined by the presence of osteoprogenitor cells and osteogenic precursor cells in the area. Both fresh autografts and bone marrow cells contain osteogenic cells, although often in decreased 


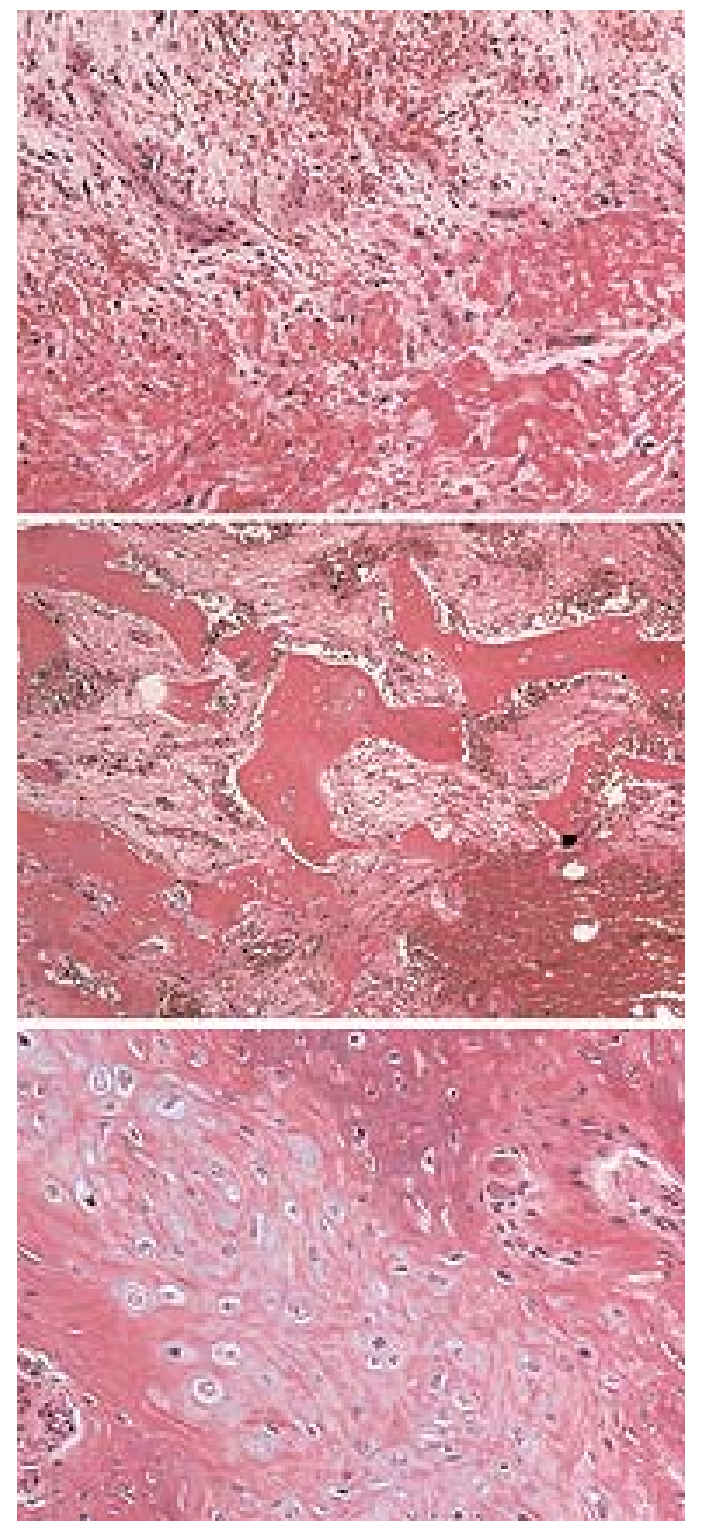

Fig. 1. Photomicrographs. Upper: Early inflammatory phase of fracture healing is characterized by organizing hematoma. This photomicrograph demonstrates ingrowth of inflammatory cells, fibroblasts, and small blood vessels into a blood clot. Note the fibrin deposition at the bottom. Center: The reparative phase consists of fibrosis and woven bone production, characterized by irregular trabeculae of immature bone and osteoid rimmed by osteoblasts, as well as reactive fibrovascular stroma. Lower: The late reparative phase is characterized clinically by hard callus formation. This photomicrograph depicts reactive cartilage (pale blue matrix) that is undergoing endochondral ossification (eosinophilic matrix) within a bridging callus. H \& E, original magnification 3100.

numbers in the elderly patient. ${ }^{22}$ Osteoconductive properties are determined by the presence of a scaffold that allows for vascular and cellular migration, attachment, and distribution. ${ }^{21}$ Osteoconduction may be achieved through the use of autografts, allografts, DBM, hydroxyapatite, and collagen. Osteoconductive properties may be altered by structure, pore size, and porosity of the scaffold. ${ }^{21}$ Osteoinduction is defined as the ability to stimulate stem cells to differentiate into mature cells through stimulation by local growth factors. ${ }^{47}$ Bone morphogenetic proteins and DBM are the most potent osteoinductive materials, although allo- and autografts have some osteoinductive properties. ${ }^{31}$

The ideal grafting material should not only be adequately osteogenic, -conductive, and -inductive but also mechanically stable and disease free. ${ }^{31}$ Autografts possess these properties; however, their use is limited by the morbidity associated with the process of obtaining them and by the often insufficient amount of graft and osteogenic precursors that are obtained. ${ }^{21}$ Allografts are osteoconductive, weakly osteoinductive, not osteogenic, and their use is recognized to have a generally higher rate of nonunion. ${ }^{4}$ This higher rate of pseudarthrosis may be the result of delayed vascular and osseous formation as well as delayed incorporation of the graft into the host site. ${ }^{4}$

Autografts and allografts may both consist of cortical or cancellous bone. Cortical bone possesses enhanced initial strength compared with cancellous bone, but over time this strength is limited by resorption secondary to the biomechanics, which may lead to resorption and the delayed incorporation of the graft. ${ }^{31}$ Cancellous bone grafts generally result in a more rapid fusion and increased delayed strength. Fresh autologous bone marrow cells may also be used in fusion to induce osteogenesis. Bone marrow cells are also osteoinductive and osteoconductive in that they have receptors for growth factors and cell adhesion molecules. ${ }^{10}$ The use of allogenic bone marrow-derived stem cells has been researched in animal models with success, although immune reactions remain problematic. ${ }^{21}$ In a clinical study, allogenic bone marrow-derived stem cells increased bone mineral content and bone deposition in three children with osteogenesis imperfecta. ${ }^{26}$ Another potential method of inducing osteogenesis is the use of stem cells predifferentiated into osteoblasts, which has been shown to lead to enhanced bone healing in animal models. ${ }^{39}$ Further development of these techniques is necessary before clinical use.

Other synthetic and natural materials have become used as scaffolds or adjuncts to scaffolds in spinal fusion including extracellular matrices, DBMs, polymers, ceramics, and the aforementioned growth factors. The goal of using these scaffolds is to induce osteogenesis through osteoconduction and to provide a delivery system for osteoinductive agents. Extracellular matrices such as collagen and glycosaminoglycans are able to aid in the differentation of osteoprogenitor cells and bind osteogenic growth factors. ${ }^{21}$ Furthermore, the chemical and mechanical properties of these matrices may be altered depending of their potential use. Demineralized bone matrix has been recognized to contain BMP, and its use in spinal fusion has been studied in animal and humans. Although initial fusion success has been demonstrated in animals studies in humans have shown autologous bone to produce higher fusion rates. ${ }^{29}$ Polymers, such as poly-glycolic acid, poly-L-lactic acid, and polylactic-co-glycolic acid, have been used in clinical studies. ${ }^{21}$ These materials are osteoconductive and are able to deliver osteoinductive factors, but their efficacy is hindered by foreign-body reactions and by mild toxicities produced during biodegradation. The ceramics HA and $\beta$-tricalcium phosphate have been studied in bone healing and found to possess a substantial 
capacity for delivering osteoinductive factors and osteogenic cells. ${ }^{24}$ Further refinement of biodegradable scaffolds will likely lead to the development of an osteoconductive and osteoinductive material that will become essential in spinal fusion.

\section{SYSTEMIC ADJUVANTS TO SPINAL FUSION}

A number of therapies including drug therapy, electrical stimulation, ultrasonography, and hyperbaric oxygen treatment have been found to enhance bone healing. As previously noted, vitamin and mineral supplements and hormone replacements lead to well-recognized enhancement of bone healing. Also frequently used in a clinical setting are bisphosphates, which have been found to increase bone mineral density and depress resorption. ${ }^{45} \mathrm{In}$ fracture settings, the bisphosphate alendroate was found to increase bone mineral density at the injured site. ${ }^{51}$

The role of electrical stimulation in spinal fusion has been increasingly researched. It has been found that direct current electrical stimulation conducted using an implantable device and external stimulation involving combined magnetic fields and pulsed electromagnetic fields increases spinal fusion rates. ${ }^{30,35}$ This success is likely the result of biochemical changes in attraction, polarity, and $\mathrm{pH}$ induced by the stimulators. ${ }^{30}$ Review of previous studies provides evidence that direct current electrical stimulation is the most effective of the modalities. ${ }^{30}$ There is evidence that combined magnetic field stimulation, which requires external stimulation of the surgical area for only $30 \mathrm{~min}$ utes/day, may enhance fusion rates. ${ }^{34}$ The authors of a recent study in rabbits provided evidence that ultrasonography may also promote spinal fusion. ${ }^{5}$ Likewise, hyperbaric oxygen therapy has been shown to improve bone healing and fusion rates in a rabbit fusion model. ${ }^{13}$

\section{CONCLUSIONS}

Optimal spinal fusion remains a critical clinical concern. To create a successful arthodesis, it is first necessary to understand the physiology of bone healing. This fundamental understanding will allow clinicians to comprehend better the unique healing process in each patient, systemically and locally. It also will promote proper use of materials with appropriate osteogenic, -conductive, and -inductive properties and of systemic adjuncts, which may be beneficial.

\section{References}

1. Alden TD, Pittman DD, Beres EJ, et al: Percutaneous spinal fusion using bone morphogenetic protein-2 gene therapy. J Neurosurg 90 (Spine 1):109-114, 1999

2. Alden TD, Pittman DD, Hankins GR, et al: In vivo endochondral bone formation using a bone morhphogenetic protein 2 adenoviral vector. Hum Gene Ther 10:2245-2253, 1999

3. Andrew JG, Hoyland JA, Freemont AJ, et al: Platelet-derived growth factor expression in normally healing human fractures. Bone 16:455-460, 1995

4. Aurori BF, Weierman RJ, Lowell HA, et al: Pseudarthrosis after spinal fusion for scoliosis. A comparison of autogeneic and allogeneic bone grafts. Clin Orthop 199:153-158, 1985

5. Aynaci O, Onder C, Piskin A, et al: The effect of ultrasound on the healing of muscle-pediculated bone graft in spinal fusion. Spine 27:1531-1535, 2002

6. Boden SD, Martin GJ II, Horton WC, et al: Laparoscopic anterior spinal arthrodesis with rhBMP-2 in a titanium interbody threaded cage. J Spinal Disord 11:95-101, 1998

7. Boden SD, Moskovitz PA, Morone MA, et al: Video-assisted lateral intertransverse process arthrodesis. Validation of a new minimally invasive lumbar spinal fusion technique in the rabbit and nonhuman primate (rhesus) models. Spine 21:2689-2697, 1996

8. Boden SD, Schimandle JH, Hutton WC, et al: In vivo evaluation of a resorbable osteoinductive composite as a graft substitute for lumbar spinal fusion. J Spinal Disord 10:1-11, 1997

9. Boden SD, Zdeblick TA, Sandhu HS, et al: The use of rhBMP2 in interbody fusion cages. Definitive evidence of osteoinduction in humans: a preliminary report. Spine 25:376-381, 2000

10. Bruder SP, Fox BS: Tissue engineering of bone. Cell based strategies. Clin Orthop 367 (Suppl):68-83, 1999

11. Burchardt H, Enneking WF: Transplantaion of bone. Surg Clin North Am 58:403-427, 1978

12. Celeste AJ, Iannazzi JA, Taylor RC, et al: Identification of transforming growth factor beta family members present in bone-inductive protein purified from bovine bone. Proc Natl Acad Sci USA 87:9843-9847, 1990

13. Chen WJ, Lai PL, Chang CH, et al: The effect of hyperbaric oxygen therapy on spinal fusion: using the model of posterolateral intertransverse fusion in rabbits. J Trauma 52:333-338, 2002

14. Copenhaver WM, Kelly DE, Wood RL: Bailey's Textbook of Histology, ed 17. Baltimore: Williams and Wilkins, 1978, pp 170-205

15. Daftari TK, Whitesides TE Jr, Heller JG, et al: Nicotine on the revascularization of bone graft. An experimental study in rabbits. Spine 19:904-911, 1994

16. Darakchiev BJ, Bulas RV, Dunsker SB: Use of calcitonin for the treatment of an odontoid fracture. Case report. J Neurosurg 93 (Spine 1): 157-160, 2000

17. DePalma AF, Rothman RH, Lewinnek GE, et al: Anterior interbody fusion for severe cervical disc degeneration. Surg Gynecol Obstet 134:755-758, 1972

18. Derynck R, Zhang Y: Intracellular signaling: the mad way to do it. Curr Biol 6:1226-1229, 1996

19. Duarte PM, Nogueira Filho GR, Sallum EA, et al: The effect of an immunosuppressive therapy and its withdrawal on bone healing around titanium implants. A histometric study in rabbits. J Periodontol 72:1391-1397, 2001

20. Fawcett DW: Bloom and Fawcett, A Textbook of Histology. New York: Chapman and Hall, 1994, pp 194-233

21. Helm GA, Dayoub H, Jane JA Jr: Bone graft substitutes for the promotion of spinal arthrodesis. Neurosurg Focus 10 (4): Article 4, 2001

22. Helm GA, Dayoub H, Jane JA Jr: Gene-based therapies for the induction of spinal fusion. Neurosurg Focus 10 (4): Article 5, 2001

23. Helm GA, Sheehan JM, Sheehan JP, et al: Utilization of type I collagen gel, demineralized bone matrix, and bone morphogenetic protein-2 to enhance autologous bone lumbar spinal fusion. J Neurosurg 86:93-100, 1997

24. Herr G, Wahl D, Kusswetter W: Osteogenic activity of bone morphogenetic protein and hydroxyapatite composite implants. Ann Chir Gynaecol Suppl 207:99-107, 1993

25. Holliger EH, Trawick RH, Boden SD, et al: Morphology of the lumbar intertransverse process fusion mass in the rabbit model: a comparison between two bone graft materials-rhBMP-2 and autograft. J Spinal Disord 9:125-128, 1996

26. Horwitz EM, Prockop DJ, Fitzpatrick LA, et al: Transplantability and therapeutic effects of bone marrow-derived mesenchymal cells in children with osteogenesis imperfecta. Nat Med 5:309-313, 1999 
27. Howard BK, Brown KR, Leach JL, et al: Osteoinduction using bone morphogenic protein in irradiated tissue. Arch Otolaryngol Head Neck Surg 124:985-988, 1998

28. Israel DI, Nove J, Kerns KM, et al: Heterodimeric bone morphogenetic proteins show enhanced activity in vitro and in vivo. Growth Factors 13:291-300, 1996

29. Jorgenson SS, Lowe TG, France J, et al: A prospective analysis of autograft versus allograft in posterolateral lumbar fusion in the same patient. A minimum of 1-year follow-up in 144 patients. Spine 19:2048-2053, 1994

30. Kahanovitz N: Electrical stimulation of spinal fusion: a scientific and clinical update. The Spine Journal 2:145-150, 2002

31. Kalfas IH: Principles of bone healing. Neurosurg Focus 10 (4): Article 1, 2001

32. Kanis JA, McCloskey EV: Effect of calcitonin on vertebral and other fractures. QJM 92:143-149, 1999

33. Kasperk $\mathrm{CH}$, Wergedal JE, Mohan $\mathrm{S}$, et al: Interactions of growth factors present in bone matrix with bone cells: effects on DNA synthesis and alkaline phosphatase. Growth Factors 3:147-158, 1990

34. Kowalski RJ, Ferrara LA, Benzel EC: Biomechanics of bone fusion. Neurosurg Focus 10 (4):Article 2, 2001

35. Linovitz RJ, Pathria M, Bernhardt M, et al: Combined magnetic fields accelerate and increase spine fusion: a double-blind, randomized, placebo controlled study. Spine 27:1383-1389, 2002

36. Marx RE, Carlson ER, Eichstaedt RM, et al: Platelet-rich plasma: growth factor enhancement for bone grafts. Oral Surg Oral Med Oral Pathol Oral Radiol Endod 85:638-646, 1998

37. Morley P, Whitfield JF, Willick GE: Parathyroid hormone: an anabolic treatment for osteoporosis. Curr Pharm Des 7: 671-687, 2001

38. Nash TJ, Howlett CR, Martin C, et al: Effect of platelet-derived growth factor on tibial osteotomies in rabbits. Bone 15: 203-208, 1994

39. Okumura M, Ohgushi H, Dohi Y, et al: Osteoblastic phenotype expression on the surface of hydroxyapatite ceramics. J Biomed Mater Res 37:122-129, 1997

40. Recker RR, Barger-Lux J: Embryology, anatomy, and microstructue of bone, in Coe FL, Favus MJ (eds): Disorders of Bone and Mineral Metabolism, ed 2. Philadelphia: Lippincott Williams and Wilkins, 2002, pp 177-198

41. Sakou T: Bone morphogenetic proteins: from basic studies to clinical approaches. Bone 22:591-603, 1998
J. G. Pilitsis, D. R. Lucas, S. R. Rengachary

42. Sandhu HS, Kanim LEA, Toth JM, et al: Experimental spinal fusion with recombinant human bone morphogenetic protein-2 without decortication of osseous elements. Spine 22: 1171-1180, 1997

43. Sawin PD, Dickman CA, Crawford NR, et al: The effects of dexamethasone on bone fusion in an experimental model of posterolateral lumbar spinal arthrodesis. J Neurosurg 94: 76-81, 2001

44. Service RF: Tissue engineers build new bone. Science 289: 1498-1500, 2000

45. Sharpe M, Noble S, Spencer CM: Alendronate: an update of its use in osteoporosis. Drugs 61:999-1039, 2001

46. Simon AM, Manigrasso MB, O'Connor JP: Cyclo-oxygenase 2 function is essential for bone fracture healing. J Bone Miner Res 17:963-76, 2002

47. Subach BR, Haid RW, Rodts GE, et al: Bone morphogenetic protein in spinal fusion: overview and clinical update. Neurosurg Focus 10 (4): Article 3, 2001

48. Tiras MB, Noyan V, Yildiz A, et al: Comparison of different treatment modalities for postmenopausal patients with osteopenia: hormone replacement therapy, calcitonin and clodronate. Climacteric 3:92-101, 2000

49. Tramontana J, Benghuzzi $\mathrm{H}$, Tucci $\mathrm{M}$, et al: Morphometric analysis of cortical bone upon the exposure to sustained delivery of anabolic promoting agents using adult male rats as a model. Biomed Sci Instrum 37:293-298, 2001

50. Urist MR: Bone: formation by autoinduction. Science 150: 893-899, 1965

51. van der Poest Clement E, Patka P, Vandormael K, et al: The effect of alendronate on bone mass after distal forearm fracture. J Bone Miner Res 15:586-593, 2000

52. Varady P, Li JZ, Cunningham M, et al: Morphologic analysis of BMP-9 gene therapy-induced osteogenesis. Human Gene Ther 12:697-710, 2001

53. Wozney JM, Rosen V, Celeste AJ, et al: Novel regulators of bone formation: molecular clones and activities. Science 242: $1528-1534,1988$

Manuscript received September 26, 2002.

Accepted in final form November 19, 2002.

Address reprint requests to: Julie G. Pilitsis, M.D., Ph.D., Department of Neurosurgery, Wayne State University, Suite 6E-UHC, 4201 Street Antoine, Detroit, Michigan 48201. email: jpilitsis@ neurosurgery.wayne.edu. 